\title{
Traumas cerebrales en niños secundarios a cabeceo de balones en fútbol. Modelo de simulación matemática
}

ERNESTO PONCE ${ }^{\text {la }}$, JESÚS PÉREZ ${ }^{2 b}$, DANIEL PONCE ${ }^{3 \mathrm{c}}, \mathrm{MAX}^{\mathrm{ANDRESEN}}{ }^{4}$

\section{Mathematical simulation of mild brain injury in children heading soccer balls}

Background: Heading professional soccer balls can generate mild traumatic brain injury in children. The long-term consequences could include difficulty in solving problems and deficits in memory and language. Aim: To assess the impact of a professional adult soccer ball on a child's head, using the finite element method and dynamic effects to predict brain damage. Material and Methods: The minimum conditions of an adult game were considered: the ball speed was $6 \mathrm{~m} / \mathrm{s}$ and the diffuse blow was 345 and 369 Newtons (N), on the forehead and top of the head, respectively. A head was modeled in order to know the stresses, strains and displacements generated by the impacts. The extent of the alteration was determined by comparing the strength of brain tissue, with predictions of computed stresses. The geometric characteristics of the head were transferred from medical images. The input data of the materials of a child's head was obtained from the literature. Results: In the case of heading with the forehead, mathematical simulation showed frontal lobe alterations, with brain stresses between 0.064 and $0.059 \mathrm{~N} / \mathrm{mm}^{2}$. When the heading was with the upper head zone, the brain alterations were in the parietal lobe, with stresses between 0.089 and $0.067 \mathrm{~N} / \mathrm{mm}^{2}$. In the cerebral spinal fluid the pressure was 3.61 to $3.24 \mathrm{~N} / \mathrm{mm}^{2}$. Conclusions: The mathematical simulations reveal evidence of brain alterations caused by a child heading adult soccer balls. The model presented is an economical and quick tool that can help predict brain damage. It demonstrates the ability of the cerebral spinal fluid (CSF) to absorb shock loads.

(Rev Med Chile 2011; 139: 1089-1096).

Key words: Brain injuries; Models, theoretical; Soccer.

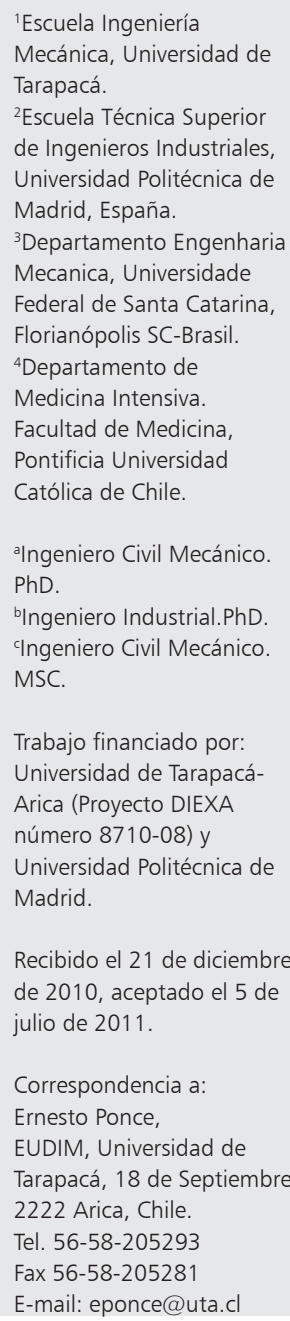

$\mathrm{E}$ studios sobre lesiones cerebrales por cabeceo de pelota en jugadores profesionales, mostraron que $80 \%$ presentaba déficits en concentración y memoria, relacionando la frecuencia del trauma con pérdida cognitiva, capacidad de planificación y disminución de percepción visual ${ }^{1,2}$.

El número de futbolistas crece $10 \%$ anualmente, lo practican unos 300 millones $^{3}$. Con esta población, se justifican estudios sobre posibles lesiones cerebrales en niños
Una investigación en 2005, sobre impactos sobre la cabeza en jugadores profesionales por golpes de codo, mano, brazo y cabeza contra cabeza (excluyendo cabeceo de pelota) indicó mayores riesgos a velocidades de $3 \mathrm{~m} / \mathrm{s}$ con un $67 \%$ de riesgo de lesión cerebral y $5 \%$ de lesión cervical $^{5}$. La magnitud de lesiones se analizó con: Head Injury Criterion, HIC ${ }^{4}$.

Otros estudios sugirieron que los daños ocurrían en duelos aéreos (32\%) y codazos $(41 \%)^{6}$. Un estudio en 33 futbolistas aficionados comparados 
con 27 deportistas de natación y atletismo, evaluados mediante los mismos test neuro-psicológicos, encontró déficits en futbolistas: Test de planeación $39 \%$ frente a $13 \%$; Test de memoria $27 \%$ frente a $7 \%{ }^{7}$.

Investigaciones sobre cabeceos revelaban atrofias cerebrales en tomografías computarizadas de jugadores en formación. Test neuro-psicológicos mostraron déficits cognitivos. Estudiando jugadores de 11,5 años promedio, aparecieron anormalidades en un $49 \%$, incluyendo dificultad para aprender nuevas palabras y dolores de cabeza ${ }^{8-10}$. En niños, una posible causa de lesiones cerebrales leves provendría del cabeceo de pelotas, ya que su sistema músculo-esquelético está en desarrollo, su capacidad para absorber golpes es limitada, tampoco tienen habilidad técnica para cabecear, porque carecen de la coordinación necesaria ${ }^{9}$.

El daño acumulativo aumentaría el riesgo de enfermedades neurodegenerativas. Como los cabeceos se hacen principalmente con la frente, las lesiones de la corteza prefrontal afectarían la capacidad de resolver problemas, memorizar, aprender y cambios en la personalidad manifestados como comportamientos impulsivos, agresivos, además depresión ${ }^{2}$.

Los modelos matemáticos, al ser comparados con la resistencia límite de los tejidos neurológicos, son una buena y cuantitativa herramienta predictiva ${ }^{11}$. Se emplean en estudios de choques automotrices y cascos protectores. Son económicos, relativamente sencillos de operar y entregan respuestas más rápidas que modelos de cadáveres de animales o seres humanos y maniquíes con sensores. En Estados Unidos de Norteamérica y Europa se busca su utilización en prevención de enfermedades como Alzheimer y Parkinson. Su finalidad principal es simular accidentes para detectar lesiones. Su nueva función es la búsqueda de pruebas en medicina forense ${ }^{12}$.

El Método de Elementos Finitos (MEF) descrito en palabras sencillas sería similar a una construcción con ladrillos, pudiéndose elaborar una obra con diferentes formas y características mecánicas según sean los tipos de ladrillos empleados. Cada elemento sería un ladrillo. Este método subdivide un volumen de material continuo en muchos elementos, que conformarían un conjunto. A cada elemento se puede dar características propias, como densidad, módulo elástico, módulo de Poisson, entre otros. Al aplicarle fuerzas exter- nas al conjunto, estas son transmitidas al interior, su efecto puede recogerse como deformaciones, tensiones e imágenes. El progreso del método en investigaciones sobre daños cerebrales, se demostró en varios trabajos ${ }^{11-14}$.

Se han comparado los criterios sobre magnitudes de lesiones cerebrales, como tensiones de Von Mises, máxima aceleración lineal, presión máxima y criterio de lesión en la cabeza (HIC) ${ }^{4}$, pero ninguno de ellos ha sido validado para niños o adolescentes. En este análisis se ha preferido el criterio de Von Mises, por ser el más conservador ${ }^{4}$.

La amortiguación en un modelo que incluyó el foramen magnum, aracnoides y cisternas, mejoró los anteriores al simular el flujo del líquido céfalo raquídeo (LCR) bajo condiciones de impacto ${ }^{15}$. Otro modelo de cabeza de adulto, incluyó cuero cabelludo, cráneo, meninges, LCR y 11 pares de venas de interconexión parasagitales, demostró que los vasos sanguíneos tienen importancia en la amortiguación de golpes ${ }^{16}$. Fueron estudiadas las meninges y la duramadre, estas inciden en atenuación de impactos debido a su deformación ${ }^{17}$. Luego el MEF pudo emplearse en prácticas forenses ${ }^{12}$. En 2009 se diseñó un modelo bio-mecánico de un niño, a escala de un modelo de cabeza-cuello de un adulto ${ }^{18}$. Últimamente se generaron modelos acoplados en niños (cabeza, cuello y tórax) ${ }^{4}$, basándose en una cabeza desarrollada en $2008^{19}$.

Desde 1961 se sabe que el cuero cabelludo absorbe veinte veces el efecto del impacto ${ }^{20}$.

El estudio de fuerzas se dificulta porque pueden emplearse distintas formas de cabecear la pelota. La intensidad del impacto depende del tipo de jugada: pase a un compañero, despeje defensivo y cabeceo al arco. El análisis de estas lesiones está en desarrollo ${ }^{21}$.

El objetivo de este estudio fue analizar el posible trauma en niños mediante el MEF, considerando efectos dinámicos para predecir daños cerebrales. La hipótesis es que se pueden localizar y cuantificar lesiones mediante un modelo matemático sencillo.

\section{Material y Método}

Se modeló el impacto de una pelota moderna, profesional para adultos, en la cabeza de un niño. Se consideraron las condiciones mínimas de juego en adultos, respecto de la velocidad de la pelota sobre la cabeza y tipo de golpe. 


\section{Características del modelo}

Las imágenes de esta investigación se basan en la experiencia de otros autores ${ }^{19,22-24}$. Se obtuvieron de imágenes médicas de tomografía computarizada, superpuestas y dibujadas en un programa para dibujo técnico (AUTOCAD). Los dibujos tridimensionales (3D) fueron exportados a un programa de MEF (ALGOR). Se emplearon elementos tipo ladrillo de 8 nodos. La geometría de la cabeza fue transformada en un volumen analítico mediante un código de malla en 3D. El modelo fue compuesto por unos 22.500 elementos y 25.000 nodos. Se consideraron cuatro diferentes tejidos: cuero cabelludo, cráneo, LCR y cerebro. Los tres primeros tejidos se modelaron como capas de igual espesor, siendo la masa cerebral el tejido interno. Como el cerebro está inmerso en el LCR, el modelo se construyó simulando que el fluido lo sostiene. Los materiales se consideraron de características isotrópicas y con un comportamiento lineal elástico en casi todos, excepto el cerebro que tiene características visco elásticas. La temperatura de referencia fue de 37 grados Celsius. El análisis se realizó en un régimen dinámico. La Tabla 1 muestra los datos de entrada.

La ecuación constitutiva del cerebro se basa en un modelo no lineal de tensión-deformación, que se desarrolla a partir de la energía de deformación, asumiendo tejido isotrópico. Fue formulada inicialmente en $1977^{25}$, luego llevada a experimentos en $1997^{26}$ y aplicada a partir de $2000^{13}$ en programas computacionales especializados. Actualmente, se puede incluir en aquellos programas comerciales que permitan modificar las ecuaciones constitutivas. Básicamente se definió como:

$$
G(t)=G(\infty))+((G(o)-G(\infty)) \exp (-\beta t)
$$

Donde:

$\mathrm{G}(\mathrm{t})=$ Módulo de cizalle

$\mathrm{G}(\mathrm{o})=$ Módulo de cizalle en corto tiempo $=$ $490 \mathrm{KPa}$

$\mathrm{G}(\infty)=$ Módulo de cizalle en largo tiempo $=$ $167 \mathrm{KPa}$

$\beta=\quad$ Constante de atenuación $=0,145 \mathrm{~m} / \mathrm{s}$

$\mathrm{t}=$ tiempo

Información obtenida de los autores ${ }^{14,25,26}$

La información fue obtenida de los autores ${ }^{19,27}$ y modificada para niños: se redujo la densidad, módulo elástico y módulo de Poisson del hueso craneal y de la piel. En el LCR se redujo el módulo de Poisson y el módulo elástico se puso algo inferior al agua de mar. Esto acorde a ensayos de laboratorio UTA.

\section{Análisis del golpe}

El impacto se consideró como carga dinámica repartida en la frente (cabeceo al arco) y en la parte superior de la cabeza (cabeceo defensivo dado en mala forma, despeje del balón).

La condición de contorno consideró que el modelo de cabeza se mueve libre respecto al cuello. El golpe es tan rápido que no resulta influenciado por las restricciones del cuello; por ello se descartan para la respuesta dinámica en un corto intervalo de tiempo ${ }^{30}$. Las restricciones se han tomado como libres en la base del cráneo, con un grado de libertad en el mismo eje del impacto. En la amortiguación de la cabeza, debe considerarse la acción protectora del cuero cabelludo; el LCR; el comportamiento del cerebro como un elastómero; los ventrículos y lagunas; la deformación del cráneo; las deformaciones de venas y arterias y otros tejidos. Es difícil conocer el factor de amortiguación real, pero es

Tabla 1. Datos de entrada para los materiales de la cabeza

\begin{tabular}{|c|c|c|c|c|c|}
\hline & Densidad & Módulo Elástico & Módulo Poisson & Módulo Cizalle & Material \\
\hline Unidades & $\mathrm{kg} / \mathrm{m}^{3}$ & $\mathrm{~N} / \mathbf{m m}^{2}$ & & $\mathrm{~N} / \mathbf{m m}^{2}$ & \\
\hline Cerebro & 1.040 & & & & \\
\hline LCR & 1.020 & 2.250 & 0,4 & 0,00002 & elástico \\
\hline Hueso & 1.200 & 8.000 & 0,2 & 3,100 & elástico \\
\hline Piel & 1.030 & 20 & 0,4 & 0,002 & elástico \\
\hline
\end{tabular}


necesario incluirlo en el análisis. Se consideró la cabeza como una estructura biológica; entonces el factor de amortiguación recomendado, en el análisis dinámico para estructuras bajo impacto ligero es $0,5^{31}$. Se consideró el mínimo de los criterios de lesión comparados en $2010^{4}$, el criterio de Von Mises: las tensiones límite para un riesgo de $50 \%$ de lesión son $0,048 \mathrm{~N} / \mathrm{mm}^{2}$ y para un riesgo de $100 \%$ de lesión son $0,080 \mathrm{~N} / \mathrm{mm}^{2}$.

La masa del balón fue de $0,43 \mathrm{~kg}$, los datos fueron tomados de FIFA quality concept for footballs ${ }^{3}$, pelota del Campeonato Mundial 2010. El tamaño es del número 5; profesional para adultos; peso de inspección entre 0,410 y 0,450 kg; presión 0,8 bares; no admite absorción de agua. La pelota de 1959 , el primer mundial de post guerra, pesaba entre 0,396 y 0,453 kg; se permitía hasta un $10 \%$ de absorción de agua ${ }^{3}$.

Datos de velocidades: Se tomaron de mediciones realizadas en 2005 en Europa considerando velocidades bajas para adultos ${ }^{32}$. La velocidad de llegada a la cabeza fue $6 \mathrm{~m} / \mathrm{s}$; la velocidad después del cabezazo (con giro de la cabeza para darle impulso) fue $7,6 \mathrm{~m} / \mathrm{s}$ y el tiempo mínimo de impacto de $14 \mathrm{~ms}$. Se analizaron dos casos:

1) Para un golpe con la frente, se consideró la condición angular para cabeceo al arco: choque en ángulo horizontal de incidencia de 9 grados y rebote 45 grados; velocidad del despeje $=7,6 \mathrm{~m} / \mathrm{s}$. Con esta información, por la teoría de impulso y cantidad de movimiento, se determinó una fuerza de $345 \mathrm{~N}$ impactando sobre la cabeza (bajo este concepto, no se precisan los coeficientes de restitución durante el impacto, ni un análisis dinámico adicional).

2) Para un impacto en la zona parietal. Una acción desafortunada, suele ocurrir en jugadores de poca experiencia. Se consideró la peor condición: un ángulo de incidencia y rebote cero grados $\mathrm{y}$ velocidad de incidencia igual a la de rebote $=6$ $\mathrm{m} / \mathrm{s}$ (al no golpear con la frente se reduciría la velocidad de rebote, porque la rotación de la cabeza tendría mínima influencia). Aplicando la teoría de impulso y cantidad de movimiento, la fuerza sobre la cabeza resulta de 369 N. En impactos de pelota sobre adultos, se han medido fuerzas de 875 $\mathrm{N}$, para máxima potencia ${ }^{2}$.

La Figura 1 (a) muestra el impacto con la frente y la Figura 3, el impacto con la parte superior de la cabeza.

\section{Resultados}

Modelo para alteraciones físicas generadas por el cabezazo con la frente

En las imágenes de resultados los rangos de colores van desde tensiones máximas (rojo) hasta mínimas (azul).

Las predicciones del desplazamiento nodal máximo fueron 0,127 a $0,114 \mathrm{~mm}$, en el cuero cabelludo. Para el desplazamiento relativo entre el lóbulo frontal y el FCR fueron de 0,013 mm, Figura 1 (b).

Las predicciones de tensiones máximas fueron 0,064 a $0,059 \mathrm{~N} / \mathrm{mm}^{2}$ en el lóbulo frontal, Figura 2 (b). Las tensiones que superaron el $50 \%$ de riesgo de lesión ocurrieron para los golpes con la frente $\left(0,048 \mathrm{~N} / \mathrm{mm}^{2}\right.$, según el criterio de Von Mises $\left.{ }^{4}\right)$. La Figura 2 (a) corresponde a las tensiones de corte en el plano de la imagen y paralelos a ella, valores máximos entre 0,010 a $0,012 \mathrm{~N} / \mathrm{mm}^{2}$.

\section{Modelo para alteraciones físicas generadas por el golpe en la zona parietal}

Las tensiones que superaron el $100 \%$ de riesgo $\left(0,080 \mathrm{~N} / \mathrm{mm}^{2}\right)$ ocurrieron para cabeceo con la parte superior de la cabeza, Figura 3.

Las predicciones del desplazamiento nodal máximo, para este modelo fueron 0,266 a 0,239 $\mathrm{mm}$, en el cuero cabelludo. Para el desplazamiento relativo entre el lóbulo frontal y el FCR fueron 0,027 mm, Figura 4 (a).

El desplazamiento nodal máximo en el cerebro ocurrió en los lóbulos frontal y parietal, 0,245 mm. El mínimo ocurrió en la base del cerebro, Figura 4 (a) y (b).

Las predicciones de tensiones máximas de Von Mises en el cerebro, fueron 0,089 a 0,067 N/mm 2 en el lóbulo parietal, Figura 5 (b). Las de corte aparecen en la Figura 5 (a) paralelas al plano de la imagen, las máximas fueron 0,033 a $0,038 \mathrm{~N} / \mathrm{mm}^{2}$

Las predicciones de tensiones máximas en el FCR, fueron 3,61 a $3,24 \mathrm{~N} / \mathrm{mm}^{2}$ en la base del cráneo (Figura 6).

\section{Discusión}

Modelo para el cabezazo con la frente

Estos datos muestran que el desplazamiento nodal, Figura 1 (b), tiene un máximo en el sector impactado: 0,266 $\mathrm{mm}$ en el cuero cabelludo. Es 


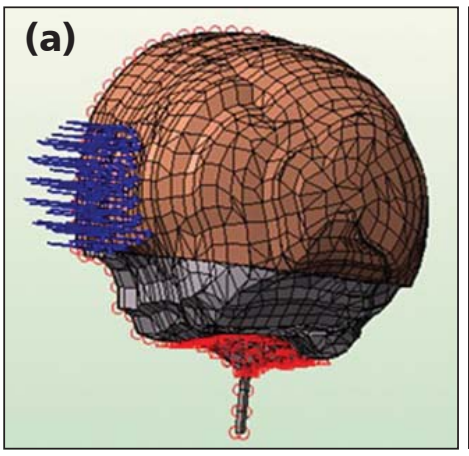

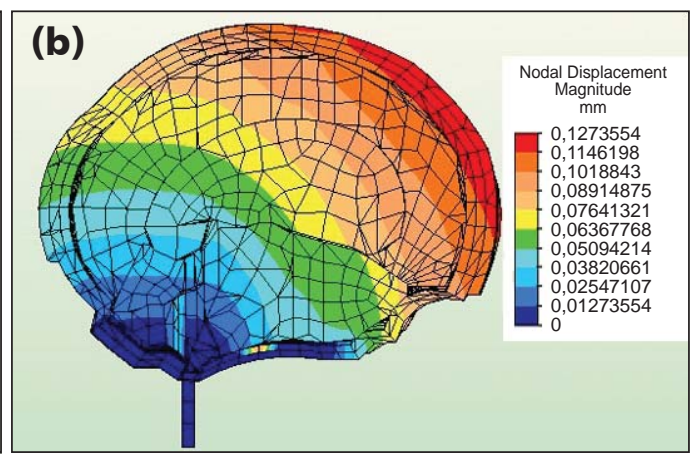

Figura 1. (a) Fuerza de $345 \mathrm{~N}$ distribuida sobre la frente, cabeceo correcto de pelota; (b) Corte sagital. Desplazamiento nodal, cabeceo con la frente.
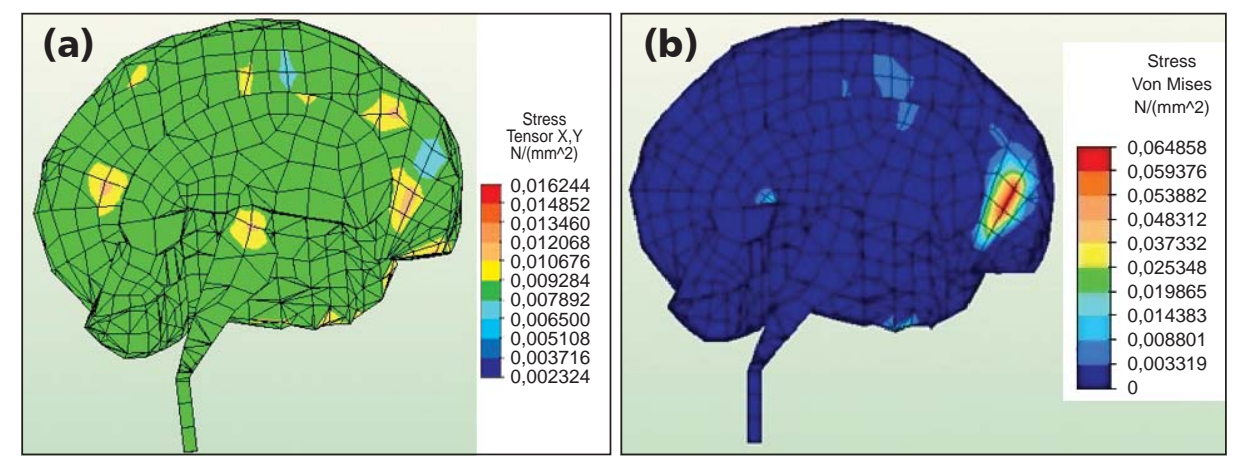

Figura 2. Corte sagital. (a) Tensiones de cizalle XY. (b) Tensiones de Von Mises debido al cabeceo con la frente.

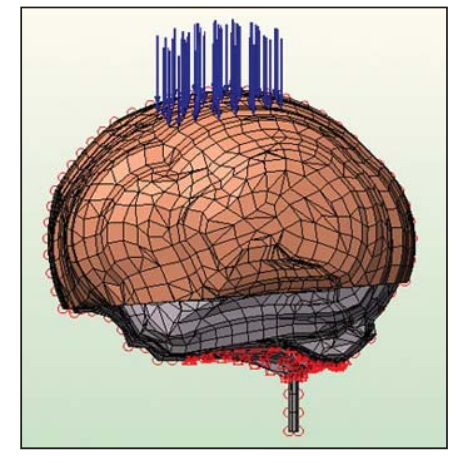

Figura 3. Fuerzas distribuidas de $369 \mathrm{~N}$ en zona parietal, cabeceo para despeje con técnica defectuosa.

razonable este resultado, la piel es más elástica que el cráneo y se desplazará sobre este con el impacto. El desplazamiento relativo entre el lóbulo frontal y FCR fue de $0,013 \mathrm{~mm}$, un valor pequeño que no conduce a grandes deformaciones ni tensiones entre aracnoides y el cerebro.

El MEF predijo tensiones entre 0,064 y 0,059 $\mathrm{N} / \mathrm{mm}^{2}$, en el lóbulo frontal. Según el criterio de Von Mises $^{4}$, el riesgo de lesión cerebral estaría sobre el $50 \%$ y bajo el $100 \%$. Inmediatamente después del cabeceo se producen las máximas tensiones de Von Mises, en una franja cercana al impacto, lo que era lo esperable en golpes no concentrados, como el de una pelota flexible. En el sector de las restricciones (base del cráneo) no aparecen grandes esfuerzos, ya que hay libertad de movimiento en dirección del choque (un criterio similar aplicado por otros autores ${ }^{30}$ ).

Figura 2 (b). Los cortantes mayores están en los lóbulos frontal y occipital, en el hipotálamo alcanzando cifras de $0,012 \mathrm{~N} / \mathrm{mm}^{2}$. Estas tensiones de cizalle suelen ser un indicativo de alteraciones físicas. Aparecen tensiones difusas en distintos lugares del cerebro, parecidas a las encontradas en análisis de otros golpes en cabezas ${ }^{33}$, geometrías coincidentes con tejidos cicatrizados difusos en autopsias de boxeadores retirados ${ }^{34}$.

La fuerza de $345 \mathrm{~N}$ impactando horizontalmente sobre la frente, se distribuye en una zona donde la sección soportante tiene mayor momento de inercia que el resto del cráneo (el momento de inercia es una medida de la capacidad para resistir fuerzas externas), también aquí el hueso presenta su mayor espesor. De acuerdo 

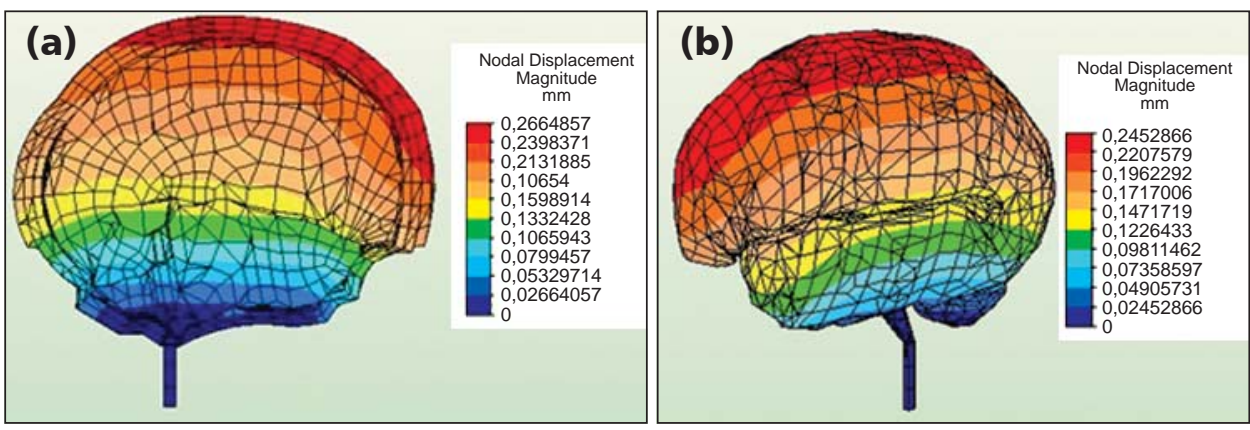

Figura 4. (a) Sección sagital. Desplazamiento nodal, despeje con zona parietal de la cabeza. (b) Vista aislada del cerebro. Desplazamiento nodal, despeje con zona parietal de la cabeza.
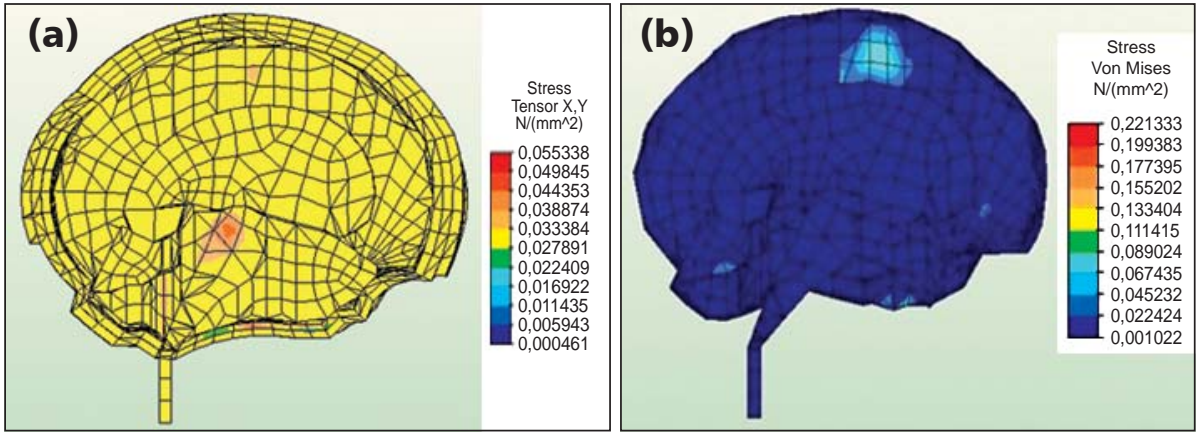

Figura 5. Despeje incorrecto con zona parietal (a) Sección sagital. Tensiones de corte $X Y$ en plano sagital. (b) Tensiones de Von Mises originadas en lóbulo parietal.

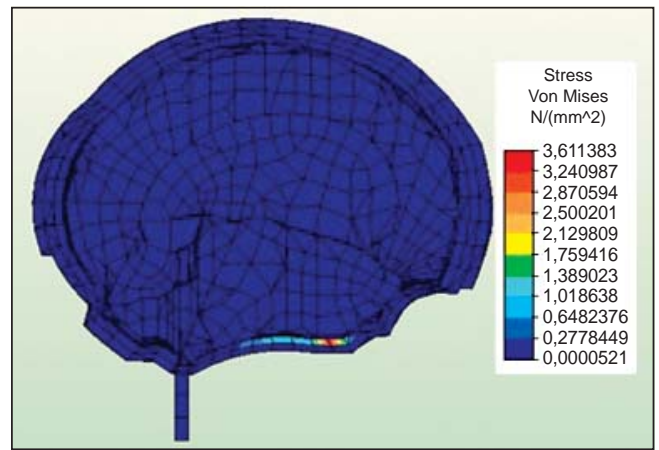

Figura 6. Sección sagital. Tensiones de Von Mises originadas en el FCR por cabezazo con parte superior de la cabeza (zona inferior entre el cráneo y cerebro).

a este planteamiento, la frente sería el lugar más resistente para cabecear.

Los resultados de este modelo indican que se pueden predecir y cuantificar teóricamente alteraciones en el cerebro, mediante el MEF y efectos dinámicos. Los modelos son simplificados, para reducir el tiempo de ejecución en el software, pero pueden ser modificados para acrecentar su complejidad.

\section{Modelo para el cabezazo con la parte superior de la cabeza}

Estos datos indican que: El desplazamiento nodal, tiene un máximo en el sector del impacto, $0,266 \mathrm{~mm}$ en el cuero cabelludo. Igual que en el caso anterior, la piel se desplaza sobre el hueso bajo el impacto. El desplazamiento relativo entre el lóbulo frontal y FCR es de $0,027 \mathrm{~mm}$, Figura 4 (a), es el doble que el golpe con la frente, siendo un indicativo de mayores deformaciones y tensiones. El desplazamiento nodal máximo del cerebro ocurre sobre el lóbulo frontal y el lóbulo parietal, estando entre 0,245 a $0,220 \mathrm{~mm}$, Figura 4 (a) y (b), que es más alto al obtenido para el cabezazo frontal, debido que golpear la pelota con esta zona ocurre bajo la peor condición de resistencia estructural del cráneo, menor espesor del hueso y mayor magnitud de la fuerza actuante $(369 \mathrm{~N})$. De acuerdo a esto, la parte superior sería el lugar menos apropiado para cabecear.

El MEF predijo tensiones entre 0,089 y 0,067 $\mathrm{N} / \mathrm{mm}^{2}$, en el lóbulo parietal, bajo el área de impacto, lo que era esperable en el inicio del cabeceo, esta zona afectada tiene dimensiones compatibles con el área de impacto Figura 5(a). Según el crite- 
rio de Von Mises, el riesgo de lesión cerebral estaría en el $100 \%$. Los cortantes tendrían un máximo de $0,038 \mathrm{~N} / \mathrm{mm}^{2}$ en la base del hipotálamo, serían tres veces mayores que en cabeceo frontal, lo que podría indicar alteraciones físicas importantes, Figura 5 (b).

En las tensiones de Von Mises y las de corte aparecen tensiones difusas, en menor cantidad que en el cabezazo frontal, debido a la dirección del golpe.

Dadas las mayores magnitudes de las fuerzas aplicadas, las tensiones deben ser mayores que en el caso de cabecear con la frente.

Se predicen tensiones elevadas en el FCR, de 3,61 a $3,24 \mathrm{~N} / \mathrm{mm}^{2}$, esto indica su capacidad de absorción de impactos. Estas tensiones ocurren entre la parte inferior del cerebro y la base del cráneo. En el líquido no hay alteraciones, porque no es tejido neurológico, sólo aumentaría su presión (Figura 6).

En los análisis para ambos tipos de cabeceo, los parámetros críticos son los diferentes módulos de elasticidad del cuero cabelludo, el hueso y el FCR, sin esas diferencias sería difícil discernir los cambios de tensiones en los distintos tejidos. En el material del cerebro es importante la ecuación constitutiva, con sus constantes de rigidez acordes al tiempo y la constante de decaimiento, lo que permite modelar un comportamiento gelatinoso.

\section{Conclusiones}

1) Se ha demostrado que es posible ubicar y cuantificar teóricamente alteraciones cerebrales originadas por el cabeceo infantil, sobre pelotas para adultos, empleando el MEF y análisis dinámico de cargas. El modelo presentado es económico y simple, siendo una herramienta rápida que puede ayudar a predecir daños cerebrales.

2) Se concluye que el modelo de cabeceo con la frente puede predecir generación de riesgos de $50 \%$ (es la técnica recomendada por los entrenadores) y el cabeceo con la parte superior de la cabeza, lo hace con $100 \%$ de riesgo (esto último sucedería por falta de control sobre la zona donde impacta la pelota, técnica defectuosa).

3) Por el cabeceo con la frente las mayores tensiones de corte ubicadas en el lóbulo frontal, podrían generar problemas en el aprendizaje; aquellas del lóbulo occipital, problemas visuales. Aparecen otras dispersas que pueden ser origen de pérdida de capacidad para memorizar y otras falencias.

4) Por el cabeceo con la parte superior de la cabeza, las mayores tensiones de corte ubicadas en la base del hipotálamo, podrían ser origen de futuros problemas conductuales.

5) También se ha validado la capacidad de absorción de impactos del FCR.

6) Las alteraciones físicas en partes de la cabeza, calculadas por la simulación, por la literatura mencionada, podrían asociarse a eventuales lesiones.

7) El modelo puede ser mejorado, al adicionar grandes vasos sanguíneos; capas de tejidos membranosos en la superficie interior del cráneo; mandíbulas y rostro, entre otros, pero aumentará el tiempo de ejecución del software.

\section{Referencias}

1. Heading the Ball in Soccer: What's the Risk of Brain Injury? Asken M, Schwartz R .The Physician and Sports Medicine1998; 26: 11.

2. The Franklin Institute on Line. The human brain. 2005 http://www.fi.edu.brain/index.htm\# [Consultado el 20 de enero 2008].

3. http://www.fifa.com [Consultado el 30 de julio 2010]

4. Meyer F, Roth S, Willinger R. Three years old child head-neck finite element modeling: simulation of the interaction with airbag in frontal and side impact. Int J of Vehicle Safety 2010; 4 (4): 285-99.

5. Withnall C, Schewchenko N, Gittens R Dvorak J. Biomechanics investigation of head impacts in football. $\mathrm{Br}$ J Sports Med 2005; 39: i49-i57.

6. Mc Clory P. Brain injury and heading in soccer. BMJ 2003; 327: 351-2.

7. Matzer E, Kessels A, Lezak M, Jordan B, Troot J. Neuropsychological Impairments in Amateurs Soccer Players. JAMA 1999; 282: 971-3.

8. Tysvaer A, Storli O, Bachen N. Soccer injuries to the brain. A neurological and electroencephalographic study of former players. Acta Neurol 1989; 80: 151-6.

9. Anderson S, Griesemet B, Johnson M, Thomas J, Martin T, McLain L, et al. Injuries in youth soccer: a subject review. Pediatrics 2000; 105: 659-61.

10. Janda D, Bir C, Cheney A. An evaluation of the cumulative effect of soccer heading in the youth population. Inj Control Saf Promot 2002; 9: 25-31.

11. Willinger R, Kang H-S, Diaw B. Tree-Dimensional 
Human Head Finite-Element Model Validation Against Two Experimental Impacts. Annals of Biomedical Engineering 1999; 27: 403-10.

12. Raul J, Deck C, Willinger R. Finite-element models of the human head and their applications in forensic practice. Int J Legal Med 2008; 122: 359-66. doi:10.1007/ s00414-008-0248-0 [Consultado el 25 de junio 2010].

13. Voo L, Kumaresan S, Pintar F, Yoganandan N, Sances A. Finite-element models of the human head. Med \& Biol Eng \& Comput 1996; 34: 375-81.

14. Belingardi G, Chiandussi G, Gaviglio I. Development and Validation of a New Finite Element Model of Human Head. Proceedings 19th ESV (Enhanced Safety on Vehicles) 2005 http://www-nrd.nhtca.dot.gov/pdf/esv/ esv19/05-0441-O.pdf [Consultado el 09 de febrero 2010].

15. Horgan T, Gilchrist M. The creation of three-dimensional finite element models for simulating head impacts biomechanics. IJ Crash 2003; 8 (4): 353-65.

16. Kleiven S, Hardy W. Correlation of an FE model of human head with local brain motion-consequences for injury predictions. In: SAE paper. Proceedings 46th Stapp Crash Conference, 2002; pp 123-44.

17. Yue X, Wang L. Viscoelastic finite element analysis of human skull-dura mater system as intercranial pressure changing. African J of Biotech Academic Journals 2008; 7 (6): 689-95.

18. Cazon A, Suescun A. A head-neck biomechanical model of 6 years old child for frontal crash studies. Int J of Vehicle Safety 2009; 4: 257-70.

19. Roth S, Vappou J, Raul J, Willinger R. Child head injury criteria investigation though numerical simulation of real world trauma. Computers Methods and Programs in Biomedicine 2008; 9(1): 32-45. Vehicle Safety 4: 257 70 .

20. Gurdjian ES, Lissner HR, Evans FG, Patrick LM, Hardy WG. Intracranial pressure and accelerations accompanying heads impacts in human cadavers. Sur Gynecol Obstret 1961; 113: 185-90.

21. Keatures C, Gregory A, and The Council on Sports Medicine and Fitness. Injuries in youth soccers. Pediatrics 2010; 125:410-414. DOI: 10.1542/peds.2009-3009; http://www.pediatrics.org/cgi/content/full/125/2/410 [Consultado el 14 de septiembre 2010].

22. Yong F, Tianzi J. Volumetric Segmentation of Brain Images Using Parallel Genetic Algorithms, IEEE Tran- sactions on Medical Imaging 2002; 21 (8): 904 -9.

23. Pitiot A, Bardinet E, Thompson P, Malandain G. Piecewise Affine Registration of Biological Images for Volume Reconstruction, Medical Image Analysis 2006; 3 (3): 465-83.

24. The Virtual Human Brain on line. Actual human brain dissection images. http://www.vh.org/adult/provider/ anatomy/BrainAnatomy/BrainAnatomy.html [Consultado el 20 de abril 2007].

25. Khalil T, Hubbard P. Parametric study of head response by finite element modeling. J of Biomechanics 1977; 10: 119-32.

26. Miller K, Chinzei K. Constitutive modeling of brain tissue: Experiment and theory 1997; 11/12: 1115-21.

27. Sklar F, Elashvili I. The pressure-volume function of brain elasticity. Physiological consideration and clinical applications, Journal of Neurosurg 1977; 47 (5): 670-9.

28. Antich P, Anderson J, Ashman R, Dowley J, González J, et al. Measurements of mechanical properties of bone material in vitro by ultrasound reflection: methodology and comparison with ultrasound transmission, Journal of Bone Miner Res 1991; 3:417-26.

29. Torres H, Zamorano M. SAR Simulation of Chiral Waves in a Head model. Rev Facing 2001; 1 (9): 3-19.

30. Chen Y, Ostoja-Starzewsky M (2010) MRI-Based Finite Element Modelling of Head Trauma: Spherically Focusing Shear Waves. Springer-Verlag. Act Mech http:// www.springerlink.com/index/y44470031t32327u.pdf [Consultado el 22 de julio 2010].

31. ALGOR (2005) Finite Element Analysis in Practice. Instructor Manual. ALGOR Inc. Pittsburg.

32. Shewchenko N, Withnall C, Keown M, Gittens R, Dvorak J. Heading in football: Part 1: Development of biomechanics methods to investigate head response. Br J Sports Med 2005; 39:i10-i25. DOI: 10.1136/ bjsm.2005.019034 [Consultado el 10 de septiembre 2010].

33. Ponce E, Ponce D. FEM 2D Analysis of Mild Traumatic Brain Injury on a Child. Lecture Notes on Computer Science. Pattern Recognition and Machine Intelligence. Springer/Berlin, vol 4815, pp 186-191. ISBN-ISSN: 03029743, DOI: 10.1007/978-3-540-77045-9 (2007)

34. The Franklin Institute on Line, The human brain (2005), http://www.fi.edu.brain/index.htm\# [Consultado en junio 2005]. 